measures of functional disability, quality of life, patient self-helplessness measure as well as the patients' adherence to therapy. Reliability and comprehensibility and sensitivity to change were also assessed.

Results: The questionnaire was assessed in 71 children with JIA, 32 with enthesitis related arthritis and 34 with cSLE. Results revealed that the developed illustrated questionnaire mean score correlated significantly with disease activity measures: JADAS-27: $r=-0.87$; ASDAS-CRP: $r=-0.85$, SLEDAI-2K: $r=-0.86$ reflecting its validity. It also correlated significantly with the scores of functional disability: $r=-0.89$, QoL: $r=-0.88$ as well as patient self-helplessness $r=-0.91$. The questionnaire was reliable (Cronbach's alpha 0.926 and had no misfitting items. The illustrations were well received and this was reflected on the questionnaire comprehensibility $(9.5)$ and sensitivity to change $(p<0.01)$. The patient motivation score showed significant $(p<0.01)$ correlation with adherence to therapy. Conclusions: The illustrated children motivation measure, is a patient-centred unidimensional scale that is valid, reliable and comprehensible. The measure has good psychometric properties indicating that it can be used at the individual child's level to tailor management and monitor changes in response to therapy. The illustrations enhanced the questionnaire perception by the children as well as the parents.

Disclosure of Interest: None declared

DOI: 10.1136/annrheumdis-2018-eular.2791

\section{THU0605 FACILITATING PATIENT CENTRED CARE: THE DEVELOPMENT OF ILLUSTRATED MULTIDIMENSIONAL PATIENT REPORTED OUTCOME MEASURE FOR CHILDREN WITH JUVENILE IDIOPATHIC ARTHRITIS}

Y. El Miedany $^{1,2}$, H. Lotfy ${ }^{3}$, N. El Aroussy ${ }^{2}$, D. Mekkawy ${ }^{2}$, S.I. Nasef ${ }^{4}$, W. Hassan ${ }^{5}$, G. El Deriny ${ }^{6}$, Y. Farag ${ }^{3}$, M. Eissa ${ }^{7}$, S. Almedany ${ }^{8}$, M. El Gaafary ${ }^{9}$, on behalf of PRINTO Egypt. ${ }^{1}$ Rheumatology, Darent Valley Hospital, Dartford, UK; ${ }^{2}$ Rheumatology and Rehabilitation, School of Medicine Ain Shams University: ${ }^{3}$ Pediatrics, School of Medicine Cairo University, Cairo; ${ }^{4}$ Rheumatology and Rehabilitation, School of Medicine Suez Canal University, Ismaillia; ${ }^{5}$ Rheumatology and Rehabilitation, School of Medicine Banha University, Banha; ${ }^{6}$ Pediatrics, School of Medicine Alexandria University, Alexandria; ${ }^{7}$ Rheumatology and Rehabilitation, School of Medicine Cairo University, Cairo; ${ }^{8}$ Rheumatology and Rehabilitation, School of Medicine Tanta University, Tanta; ${ }^{9}$ Community and Public Health, School of Medicine Ain Shams University, Cairo, Egypt

Background: The advances in paediatric rheumatology management have mandated a drastic change in the way children with juvenile arthritis are assessed and monitored. As a consequence, there has been a call for new outcome measures that reflect a more holistic approach to day to day standard management. Such an emphasis reflects contemporary views about the relation between mind and body, and acknowledges the critical link between physical and psychological health as well as adherence to therapy amongst the children living with inflammatory arthritis.

Objectives: To assess validity; reliability and responsiveness to change of an illustrated child/parent Multidimensional Patient Reported Outcome Measures questionnaire which can assess construct outcome measures of children with juvenile inflammatory arthritis.

Methods: 106 children with juvenile inflammatory arthritis were included in this work in a multicentre study. The questionnaire was developed by integrating information obtained from children living with JIA as well as their parents. The questionnaire included 5 main categories which are patient-centred: Health related quality of life: functional ability (children health assessment questionnaire) and quality of life (10-items reflecting psychological, social, school and behavioural issues as well as the patient's own perception). 2. Disease activity measure: pain intensity, the child's overall well-being, measure of fatigue and morning stiffness using (0-10 numerical visual analogue scale), 3. self-reported joint tenderness and swelling. 4. Current medication, side effects as well as adherence to therapy (2 questions using 0-10 numerical visual analogue scale); 4. Comorbidities as well as 5 . Patient motivation. All the items were supported by illustrations to explain the question and make it easier to understand. The questionnaire has parent and patient versions. The disease activity status was assessed using JADAS-27.

Results: The questionnaire was reliable as demonstrated by a high-standardised alpha $(0.890-0.978)$. The questionnaire items correlated significantly $(p<0.01)$ with clinical parameters of disease activity. The patient reported tender joints correlated significantly with the physician's scores $(0.842)$. Changes in functional disability, quality of life as well as the motivation score showed significant variation $(p<0.01)$ with diseases activity status in response to therapy. The illustrated PROMs questionnaire showed also a high degree of comprehensibility (9.6).

Conclusions: Integrating patient reported outcome measures into standard clinical practice is feasible and applicable. This version of illustrated multidimensional questionnaire was found to be valid and reliable. It provides informative quantitative measure for the disease activity core set data, and in the meantime, facilitates assessing the children's health related quality of life measure, adherence to therapy, comorbidities as well as motivation on individual basis.

Disclosure of Interest: None declared

DOI: 10.1136/annrheumdis-2018-eular.2789

\section{THURSDAY, 14 JUNE 2018 \\ Other orphan diseases}

\section{THU0606 \\ EFFECT OF INTERLEUKIN-1 ANTAGONISTS ON THE QUALITY OF LIFE IN FAMILIAL MEDITERRANEAN FEVER PATIENTS}

Ö. Varan ${ }^{1}$, H. Küçük ${ }^{2}$, H. Babaoğlu' ${ }^{1}$, N. Atas ${ }^{1}$, H. Satıs ${ }^{1}$, R. Bilici Salman ${ }^{1}$, A. Tufan ${ }^{1}$, B. Göker ${ }^{1}$, S. Haznedaroğlu' ${ }^{1}$, M.A. Öztürk ${ }^{1} .{ }^{1}$ Rheumatology, Gazi University, Ankara; ${ }^{2}$ Rheumatology, Education and Training Hospital, erzurum, Turkey

Background: Familial Mediterranean Fever (FMF) is characterised by febrile inflammatory painful attacks of peritonitis, pleuritis, arthritis and erzypeloid erythema. Colchicine is the mainstay of treatment in FMF but about\%10 patients do not respond well to colchicine. IL-1 antagonists have been shown to be effective in the prevention of attacks in colchicine resistant FMF (crFMF) patients. Herein we investigated effect of these agents on quality of life of crFMF patients.

Objectives: to investigate effect of IL-1 antagonists on quality of life of crFMF patients.

Methods: Data is derived from Gazi FMF cohort which was established in year 2010. Since then data of patients with FMF who were diagnosed according to the Tel Hashomer criteria were registered. Co-morbidities, detailed attack characteristics, treatments, laboratory parameters and impact of FMF on their life in terms of quality of life were recorded. A retrospective cohort analysis was made from records of patients who were treated with IL-1 inhibitors. SF-36 form was filled before and 3 months after the IL-1 antagonist treatment. Wilcoxon test was used for the analyses and a p value equal or less than 0.05 is considered as statistically significant.

Results: there were 41 patients ( 24 women and 17 men). Anakinra was used in 33 patients and 8 patients received canakimumab. There was a statistically significant reduction in the frequency, severity and the duration of attacks after treatment with IL-1 antagonists ( $\mathrm{p}<0.001$, for each). A statistically significant improvement was observed in all domains of SF-36 (figure 1).

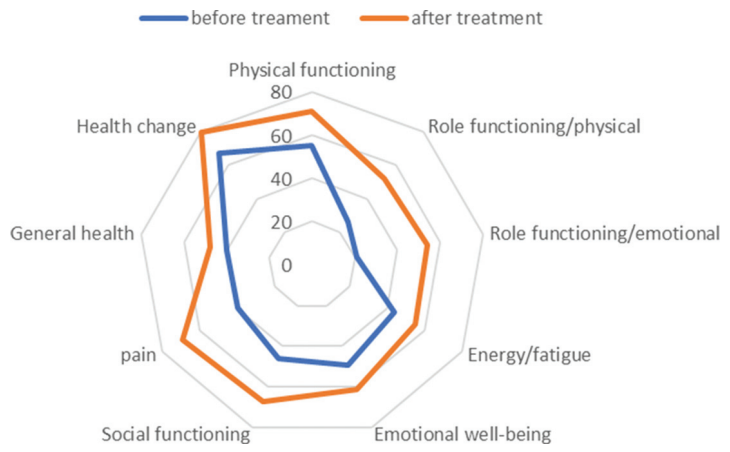

Figure. The change of SF-36 parameters before and after treatment

Conclusions: IL-1 inhibitor therapy reduces frequency, severity and duration of attacks and significantly improves the quality of life of crFMF patients.

Disclosure of Interest: None declared

DOI: 10.1136/annrheumdis-2018-eular.6495

\section{THU0607 AUTOINFLAMMATORY SYNDROMES: CLINICAL AND GENETIC CHARACTERISATION OF A COHORT OF ADULT PATIENTS IN A SINGLE REFERENCE CENTRE}

A. Ruiz Roman ${ }^{1}$, S. Garcia Morillo², M.A. Montes Cano ${ }^{3}$, M. Lisbona Muñoz ${ }^{1}$ C. Aguilera Cros ${ }^{1}$, A. Muñoz Jimenez ${ }^{1}$, E. Rubio Romero ${ }^{1}{ }^{1}$ Reumatologia; ${ }^{2}$ Medicina Interna; ${ }^{3}$ Inmunologia, Hospital Universitario Virgen del Rocio, Sevilla, Spain

Background: Autoinflammatory syndromes are diseases that are characterised by increased inflammation mediated by cells and molecules of the innate immune system. Currently, new techniques have been developed that allow earlier diagno sis and treatment of these pathologies. 